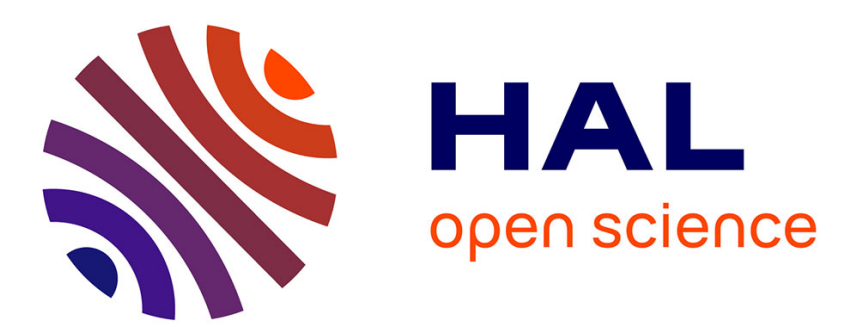

\title{
Dynamic consistency problems behind the Kyoto protocol
}

Minh Ha-Duong, Jean Charles Hourcade, Franck Lecocq

\section{To cite this version:}

Minh Ha-Duong, Jean Charles Hourcade, Franck Lecocq. Dynamic consistency problems behind the Kyoto protocol. International Journal of Environment and Pollution, 1999, 11 (4), pp.426-446. halshs-00002459

\section{HAL Id: halshs-00002459 \\ https://shs.hal.science/halshs-00002459}

Submitted on 5 Aug 2004

HAL is a multi-disciplinary open access archive for the deposit and dissemination of scientific research documents, whether they are published or not. The documents may come from teaching and research institutions in France or abroad, or from public or private research centers.
L'archive ouverte pluridisciplinaire HAL, est destinée au dépôt et à la diffusion de documents scientifiques de niveau recherche, publiés ou non, émanant des établissements d'enseignement et de recherche français ou étrangers, des laboratoires publics ou privés. 


\title{
Dynamic Consistency Problems behind the Kyoto Protocol
}

\author{
Minh Ha-Duong Jean-Charles Hourcade Franck Lecocq*
}

November 2, 1998

\begin{abstract}
This paper examines the economic rationale behind both the quantitative targets and the flexibility mechanisms adopted in the Kyoto Protocol. It synthesises some theoretical dimensions of the debate about the so-called "when flexibility" of climate policies, explaining the importance of the interplay between uncertainty and technicoeconomic inertia. Numerical results shows that the aggregate Kyoto abatement target is consistent with a stochastic dynamic optimum in which a $450 \mathrm{ppm}$ concentration ceiling is seriously considered.

Turning to the EU-US debate about the interpretation of the "supplemental to" condition in Article 3 of the Kyoto Protocol regarding the articulation between international trading systems and domestic policies and measures, this text illuminates the risk of dynamic inconsistencies due to the heterogeneity of capital stocks in the economy, if price signals do not emerge in due time from GHGs trading systems because of the "hot air" in some countries and the discovery of low costs abatement potentials in Annex B countries.

Numerical simulations show that a delay of action on sectors with large inertia of capital stocks and of the consumption styles may under such circumstances ultimately undermine the economic viability of climate policies beyond 2012. Some lessons are derived for the future of climate policies and negotiations about the implementation of the Kyoto Protocol.
\end{abstract}

\section{Introduction}

The most heated scientific debates before the Kyoto UN Framework Convention on Climate Change (FCCC) third Conference Of Parties (UNF 1992) focused about the so-called when and where flexibility

\footnotetext{
${ }^{*}$ CIRED (Centre International de Recherche sur l'Environnement et le Développement), Campus du Jardin Tropical, 45bis avenue de la Belle Gabrielle, 94736 Nogent sur Marne CEDEX, France. Ha Duong acknowledges support from EC contract No.ENV4 - CT96 - 0197, Lecocq from ISI project "Shared Analysis".
} 
as pre-conditions for cost-effective policies aiming at stabilising greenhouse gases (GHGs) concentration over the long run. The "where flexibility" argument underpinned the demand by many OECD countries to include various forms of international GHGs emissions trading in the Protocol to guarantee that emission abatements will occur where they are less expensive. The "when flexibility" argument was critical in discussions about what global level of abatement in 2010 should be the economically and environmentally founded outcome of the negotiation.

The "when flexibility" debate has raised many important theoretical questions. To pretend that the Kyoto conference has been driven by economic rationality would be difficult. Its outcome was indeed greatly influenced by the political and diplomatic process. Still, it is worth elucidating to what implicit assumptions and value judgments the agreement achieved in December 1997 corresponds to.

We will examine in a first step the economic rationale behind the quantitative targets of the Kyoto Protocol. Our goal in that section will be to clarify why the main decision parameters to be considered in a collective optimum perspective differ when one passes from a deterministic decision-making framework to a stochastic decision-making framework. This will lead us to define six theoretical dimensions of the timing debate: discounting, inertia, technical change, irreversibility effect, dependent learning and risk aversion.

In the second section we will examine the Kyoto Protocol in a public economy perspective. Regarding the "when flexibility", we will utilise results from the dynamic optimisation model DIAM (Ha-Duong, Grubb \& Hourcade 1997) to demonstrate that Kyoto targets are more ambitious than those figured out in the Wigley, Richels \& Edmonds's (1996) Nature paper. Actually they are consistent with a stochastic dynamic optimization analysis in which a $450 \mathrm{ppm}$ concentration ceiling is considered with a rather high level of subjective probability. Regarding "where flexibility" mechanisms, we will explore the economic rationale behind the EU-US debate about the interpretation of the Kyoto Protocol Article 3 "supplemental to" condition in regarding the articulation between international trading systems and domestic policies and measures.

We will interpret this debate as underpinned by a dynamic consistency problem; if indeed, because of the availability of excess allocation of emissions rights (the so-called "hot air") in countries such as Russia and Ukraine and because of the discovery of important negative cost potentials in Annex B countries, international price of carbon remain low over the following decade (as suggested by the most influential energy forecasting models), then to rely on the sole price signals of the emissions trading may not allow for minimising the overall costs of climate policies over the long run. Low price signals may indeed be not strong enough to trigger in due time decisions apt to curb down emissions trends in sectors such as transportation and housing, which would make very difficult for some countries to adopt more ambitious emissions reduction targets after the first budget period of the Protocol (2012). 
In a third step we will demonstrate the order of magnitude of this dynamic consistency problems by considering the heterogeneity of capital stocks in the START model which is a bi-sectoral dynamic optimisation model. It will be shown that a delay of action, i.e. a delay in policies and measures beyond the sole response to short term pricesignals, in a sector such as transportation which is characterised by a lower price response and a higher inertia than the industrial or the energy sectors, may ultimately be proven so costly that the long run FCCC objective may be undermined.

\section{Theoretical background of the timing de- bate}

\section{1 $\quad \mathrm{CO}_{2}$ ceilings and emissions}

Azar (1998) recently highlighted the policy issues behind the 'when flexibility' debate by demonstrating the short-term consequences of various long-term $\mathrm{CO}_{2}$ concentration ceilings:

- Setting such a ceiling at 650 parts per million (ppm) is consistent with global $\mathrm{CO}_{2}$ emissions averaging around 10 Gigatons of Carbon $(\mathrm{GtC})$ per year during the next century. Given that current emission level is around $7 \mathrm{GtC}$ (more or less $1 \mathrm{GtC}$ ), there is no sense of urgency for this target.

- Achieving $450 \mathrm{ppm}$ is consistent with an average carbon budget of about $6.5 \mathrm{GtC}$ per year during the next century. Little explanations is needed to understand that immediate action is required to ensure compatibility between this target and future demographic and economic growth.

- For $550 \mathrm{ppm}$, a careful analysis of arguments is required. To better understand the implications of this $550 \mathrm{ppm}$ concentration ceiling will then be the first motivation for the analysis undertaken in this section.

Yet however useful and convenient cost-constraints analysis may be, ultimately the choice of any target will depend upon the relative comparison of its costs versus its benefits. From this point of view, analysing the economics of greenhouse gases mitigation, even if it deals only with one half of the issue, is a necessity for the choice of any concentration limit.

Clearly enough, the uncertainty about the damages is the most difficult issue in climate change economics, as it leads to critical problems in estimating benefits of action. Nobody knows what GHGs concentration level would really be consistent with a sustainable development perspective and that the costs of technical responses could be realistically anticipated.

Still it is possible to distinguish three parameters that would remains to determine the balance between an early or belated GHGs 
abatement even with perfect information today about the long term target. These are discounting, inertia and technical change.

But their impact is affected by the consideration of various forms of uncertainty in relation to the three remaining aspects which are to be considered in a stochastic setting: irreversibility effect, dependent learning and risk aversion. The technical and theoretical difficulties in dealing with these parameters in the analysis should not hide their importance in real decision-making situations. Indeed, public debates about the appropriate timing of action for global $\mathrm{CO}_{2}$ emissions reduction has been and remains paradoxically blurred by the lack of consideration or by a misunderstanding of the nature and role of uncertainties surrounding climate issues and policies.

\subsection{Key parameters in a deterministic analysis}

Let us adopt temporarily the usual deterministic decision-making framework. This is equivalent to assuming that we are certain of what constitutes dangerous anthropogenic interference with the climate system and what needs to be done to prevent it. Another justification for this framework could be that, while acknowledging uncertainties, we select normatively a greenhouses gases stabilisation level once for all. In such a setting discounting, inertia of economic systems and technical change justify that early abatement may be proved less cost effective than abatements postponed to further time periods, at least for targets above $550 \mathrm{ppm}$ ((Wigley et al. 1996)).

Discounting Climate policies will have consequences upon both the present and future generations and any decision comes practically to a form of implicit or explicit weighting of the value of events occurring at different points in time. Economic analysis try and make this weighting explicit by using a discounted utility function for comparing different inter-temporal welfare distributions. This makes the welfare losses of given abatement costs lower in the future, and, consequently, a higher discount rate makes postponing action more attractive.

This raises the question of the appropriate level of the discount rate. Debates in this field have been marked by a recurrent opposition between those who argue in favour of a $8-10 \%$ discount rate which reflects market interest rates and those who support a $0 \%$ discount rate. Interestingly however this burning debate can be calmed down if one comes back to basic principles of economic analysis (see Chapter 2 and 4 in (Bruce, Lee \& Haites 1996)) :

- A fundamental justification for discounting is the argument that, given the economic growth observed over the last two centuries, future generations are likely to be much richer than we are. This argument, strongly supported by T. Schelling (Schelling 1995), suggests that the cost of climate change mitigation policies will impose a sacrifice on the poorer (present) generation and will increase the well being of the richer (future) populations. 
- When long term time profiles are considered the level of interest rates on financial markets cannot be a good indicator for the social discount rate; only the return rate on risk-free public long term investments has to be considered, which represents the long term marginal efficiency of investments in a given economy. This rules out the use of a 8-10\% discount rate except for some developing economies for a few decades.

- One can justify, in a normative approach, a null pure time preference rate. But in addition to pure time preference, the discount rate still encompasses the effect of increasingwealth-and-decreasing-marginal-utility. This effect is measured by the elasticity of the marginal utility of consumption multiplied by the rate of economic growth. Thus, a null discount rate comes to assume that the marginal productivity of capital is zero (in utility terms) which is in turn unrealistic.

This is why, for developed economies, a descriptive approach suggests a long term discount rate around $5 \%$ when a normative approach with a $1 \%$ time preference rate would lead to retain between 2 and $3 \%$. Within these orders of magnitude, the quantitative impact of the discount rate is principally conditional upon the decision-making framework utilised:

- In a cost-efficiency framework where the aim is to minimise the costs of meeting a given GHGs concentration ceiling, dynamic analysis demonstrates that, in first best setting, the shadow value of this ceiling increases yearly as the discount rate. The delay in abatement imposed by the selection of a high discount rate is then in part mitigated by this form of Hotelling rule; the ceiling is indeed an exogenous normative constraint which expresses the 'benefit side' of climate policy in a form which is irrespective of the level of the discount rate.

- In a cost-benefit analysis framework discounting has conversely a very strong effect on this 'benefit' side. Indeed, climatic damages occur so far in the future that even rather modest divergences in the discount rate may change significantly their present value and influence the timing of action; in fact the key parameter is not the discount rate as such: what matters is whether the rate of increase of the value of climatic damages relative to growth is high enough to compensate the impact of the discount rate.

Inertia Socio-economic systems are clearly characterised by important inertia in the sense that rapid changes of their evolution require far larger amount of efforts than smooth adaptations. In particular most economic analysts pointed out the fact that accelerating the turnover of capital stocks would imply higher costs of climate policies because the costs of premature retirement of existing capital stocks are to be covered in addition to the costs of abatement techniques. 
But inertia has also an opposite effect: the more important is the inertia (it is reasonable to anticipate that reforming the energy systems will take at least 50 years) the sooner one has to start. This is why the IPCC (IPCC 1995) states:

The choice of abatement paths involves balancing the economic risks of rapid abatement now (that premature capital stock retirement will later be proven unnecessary), against the corresponding risks of delay (that more rapid reduction will then be required, necessitating premature retirement of future capital).

The balance between both these effects is a matter of empirical evaluation, but in a certainty case it could be argued that, given a $550 \mathrm{ppm}$ target, the balance might not be very much tilted in favour of early abatement.

Recent debates on economic and social inertia have extended beyond the question of physical capital stock turnover which spans from 5 to 50 years, depending upon the type of equipment considered. They led to the idea that part of the emissions dynamics is determined by parameters beyond the energy sector and whose inertia may be far higher. Mark Jaccard (Jaccard 1997) portrays the great diversity of the sources of inertia by a three level hierarchy of the decisions governing the dynamics of emissions and energy demand:

The end use equipment: For the selection of equipment using energy in a more or less efficient way, decisions are made by private agents and the turnover of capital stock ranges from a few years to two decades. At this level the relative cost of delivering a given energy service is the key criteria within informational constraints and market imperfections inhibiting the access to the best available technologies.

The infrastructure and industrial equipments: This level is largely governed by centralised public and/or private decisionmakers. It encompasses the buildings, the major transit modes, and industrial infrastructure. The turnover of capital stocks is measured in decades and every decision involves an amount of capital whose magnitude is far higher than at the end-use level. Except in some energy intensive activities, energy costs is a minor decision parameter compared with, for example, strategic criteria in the industry or cost/speed ratio in the transportation sector.

Land-use and urban planning: this level is driven by infrastructure decisions and by public policies which can either be explicit, i.e. urban planning, incentives to an even distribution of the human settlements, or implicit, i.e. subsidies to mobility, or rules governing tenants and landlords relationships. It determines greatly the growth of transportation needs and related demand for fuels.

Beyond the turnover of capital stocks, inertia in the economic 
system results from the interactions between these three levels. Final energy demand is driven not only by the efficiency of the end-use equipment but also by structural changes in the production sectors (share of energy intensive industries or as just-in-time production; processes) and by evolutions in life styles and in geographical distribution of human settlements.

For example, the very architecture of the buildings determines the air conditioning requirements; urban forms determine not only the transportation needs but also the relative share of travels made on foot, on bicycles, by rail or by private car. The attraction of activities around the proximity of infrastructures, the induced investment, the nature of skills and the amount of embedded interests generate dynamics which are hard to curve overnight.

Technical change The third and last parameter that supports the idea of belated GHG abatements is technical change on carbon saving techniques. If, thanks to invention and adoption of technical innovation, costs of these techniques decrease along with time, then technical progress concurs with discounting: it decreases the relative cost of future efforts.

But the fact that most available modeling tools capture this process through an autonomous technical change coefficient may reinforce the common misperception that carbon saving technical change is a "Manna from Heaven" whose quantity steadily grows over time. Considering more realistically the fact that technical progress is yielded by investments in research and development leads to a different view because it focuses on the timing of required policy signals.

This is why, even from rather different perspectives, Grubb (Grubb 1997) as well as Schneider and Goulder (Schneider \& Goulder 1997) point out the conceptual distinction to be made between abatement investments within a given technical endowment and policy action, such as a carbon tax aiming primarily to induce low-cost alternatives in the future.

This logical distinction between the timing of abatement and the timing of action is even more important in the uncertainty case as will be demonstrated hereafter.

These three dimensions led Wigley, Richels and Edmonds (Wigley et al. 1996) to point out that the least cost abatement path towards a $550 \mathrm{ppm}$ concentration target should be only a few percent below the baseline emissions in 2020 if the target is certain.

\subsection{Key parameters in a stochastic framework}

Reasoning in a certainty case (how to minimise costs of meeting a given target, or how to minimise the total cost of the climate policies) comes to ignore that, despite current progress in climate sciences, we are not likely to know in the near future at what concentration level 
'dangerous interferences with the climate system' would occur, which is the FCCC objective adopted at Rio in 1992. It also ignores other uncertainties, endogenous to human behaviours, which may influence the timing of action:

- The range in the estimates of climate sensitivity ${ }^{1}$, assessed in (IPCC, Working Group I 1997) is $+1.5^{\circ} \mathrm{C}$ to $+4.5^{\circ} \mathrm{C}$. Moreover, much remains to be discovered in the future on specific issues such as the west Antarctic ice sheet, oceanic thermohaline circulation in north Atlantic or the El Niño-Southern Oscillation, even if knowledge has accumulated at an impressive rate over the last decade,

- Sudden changes in public concern: past experience demonstrates that political life cycles of environmental issues is not only driven by scientific discoveries or symptomatic events, but also by the necessary maturation of the public acceptance of new risks, by possible mismanagement of information, (e.g. the "mad cow" crisis) or by the combination of political parameters as illustrated by the Waldsterben crisis example.

- Evolutions in energy demand and technology are intrinsically uncertain. Most of baselines retained in recent forecasting studies incorporate expectations of stable or steadily increasing energy prices over the following decades. But these are not fully supported by recent analysis of structural determinants of oil prices, which underlines in particular the drastic decrease of the cost of new discoveries (Fagan 1997); moreover they do not capture possible bifurcations in trends in the transportation sector over the long run, which are conditional upon today infrastructure decisions.

This is why, "The challenge is not to find the best policy today for the next 100 years, but to select a prudent strategy and to adjust it over time in the light of new information." This statement from (Bruce et al. 1996), however banal it may look, forces to account for three additional parameters in public decision:

\section{Environmental and Technological Irreversibility Effects The FCCC}

considers implicitly that a "safety ceiling" for greenhouse gases concentration exists over which non acceptable damages may occur. Without uncertainty about the value of this safety ceiling, the physical irreversibility could simply be treated as a technical constraint among others in an optimisation program. The economic irreversibility effect arises from the facts that such a ceiling cannot be specified today and that delaying action means giving up the option of reaching the lowest stabilisation levels because of the physical accumulation of greenhouse gases in the atmosphere at least at the human generation time-scale.

\footnotetext{
${ }^{1}$ The long term (equilibrium) change in global mean surface temperature following a doubling of atmospheric equivalent $\mathrm{CO}_{2}$ concentration, noted $\Delta T_{2 x}$.
} 
The combination of this environmental irreversibility with unexpected bad news from climate science could consequently lead to a sudden acceleration of adaptation and mitigation policies to compensate a delay in abatement efforts. For this reason, stabilisation of $\mathrm{CO}_{2}$ concentration at $400 \mathrm{ppm}$ has already become an goal difficult to defend in a full cost-benefit analysis (although is can be noted that there exists several consistent global energy scenarios to this target). It will be the same for $450 \mathrm{ppm}$ in a couple of decades if present emissions trends continue.

This confirms the existence of a "window of opportunity" for any concentration target ((Hourcade \& Chapuis 1995)). Out of this window of opportunity, we would then face the dilemma of choosing between economically disruptive policy measures, or face climatic changes which are today viewed as unacceptable. An earlier mitigation action may increase flexibility in moving toward stabilisation of atmospheric concentrations of greenhouse gases.

But the reverse is also true. It is still arguable that, ultimately, damages due to climate change will be proven negligible even for an average temperature increase well over $2^{\circ} \mathrm{C}$. Then, symmetrically to the environmental irreversibility effect an investment and technical irreversibility effect has to be considered, which (Dixit \& Pindyck 1994, esp. pp. 412-418) sets a brake to climate change mitigation policies. It implies that waiting for more information will avoid the risk of over-protecting the environment.

The balance of these two opposed irreversibility effects is still an unsettled issue. Here we would like to add to those debates explored recently in (Ha-Duong 1998b, Narain \& Fisher 1998), the idea that conclusions may depend significantly on the treatment of technical change. If indeed, instead of being viewed as "autonomous" or induced in a very flexible manner by public policies, technical change is treated as an autocatalytic process of learning-by-doing, economies of scale, informational increasing returns and positive network externalities, then it can induce bifurcations and "lock-in" processes (Arthur 1989).

Beyond a critical point, market forces tend indeed to reinforce the first choice in a self-fulfilling process instead of correcting it (Hourcade 1993). Seen from 1998, there are several possible market equilibria in 2020, and several possible states of the world characterised by different technical contents. The bifurcation towards one or another depends upon the early decisions made today and on the present expectations.

For example, we can easily distinguish two very different equilibria in the transportation sector with relatively similar total costs but very different carbon contents: they can't be discriminated today but the costs of shifting from the adopted one to the other in the future might be all the more important that the transition period is short. In such a setting the technical irreversibility effect may be higher than generally expected in literature. 
Dependent learning Another important component of option value in the context of global environmental risks has been named dependent learning in (Fisher \& Hanemann 1987):

It surely requires no algebra to show that, if the information about the consequences of an irreversible development action can be obtained only by undertaking development, this strengthens the case for some development.

Here again one has to consider symmetrically this effect on the environmental side and on the technological side (see (Mégie 1992) for the case of the ozone layer and CFC phasing out). Less $\mathrm{CO}_{2}$ emissions would slow the rise of the climate change 'signal' over the climatic natural variability 'noise'. But this effect, which supports the idea of more emissions over the short run (Kolstad 1994), may actually be very small. According to (Bolin 1998), implementing the Kyoto protocol would only make a difference of 1 or $1.5 \mathrm{ppm}$ for the $\mathrm{CO}_{2}$ concentration in 2010, which is to be compared with the about $120 \mathrm{ppm}$ increase over the preindustrial level.

Conversely, emission control policies are likely to bring significant scientific, technical and institutional learning. This is why in the case of climate change we argue that the dependent learning effect is far higher on the technology-side. This is an argument for earlier decisions which may not have received full attention in climate policy models to date.

Risk aversion The preceding sentence applies even more to the risk aversion parameter. Table 1 shows risk aversion as an argument for early action against climate change, which confirms common wisdom. Indeed, one might argue that informed societies should not be indifferent to global risks even if their probability is low: which citizen would allow its government for playing roulette with the budget, even with a small net positive expected gain ?

To introduce risk aversion in the timing debate require to go beyond the classical maximisation of an inter-temporal utility and use the Kreps-Porteus preferences illustrated equation 1:

$$
J\left(c_{1}, \tilde{c_{2}}\right)=u\left(c_{1}\right)+\beta u\left(v^{-1}\left(\mathrm{E} v\left(\tilde{c_{2}}\right)\right)\right)
$$

This function $J$ expresses the preferences of a decision maker living two-periods, facing a certain gain $c_{1}$ in period one and a stochastic gain $\tilde{c_{2}}$ in period two. In this equation, $\beta$ is the discount factor, $v^{-1}\left(\mathrm{E} v\left(\tilde{c_{2}}\right)\right)$ is the certainty equivalent ${ }^{2}$ of $\tilde{c_{2}}$ for an utility function $v$, and $u$ is the felicity function. The point we would like to make here is that almost all previous modeling work equals the rate of resistance to inter-temporal substitution $-u^{\prime \prime} / u^{\prime}$ with the rate of absolute risk aversion $-v^{\prime \prime} / v^{\prime}$ by having $u=v$, and thus is focused on a rather special case for which little 
justification can be found.

There are different reasons explaining the difficulty of accounting for pure risk aversion in the timing debate. First, this collective decision raises ethical questions on how to justify any specific utility function. Second, there are some real technical problems to solve integrated assessment models in stochastic mode, especially if one try to account for all the points discussed above which lead to large and non convex problems.

These, along with deeper conceptual concerns on endogenous uncertainties, may explain why some analysts such as Alcamo (1994) or Toth, Bruckner, Füssel, Leimbach \& Petschel-Held (1997) have chosen to do without any subjective probabilities and abandoned the Bayesian framework. Yet this does not rules out the logical necessity of considering risk-aversion as a factual economic behaviour.

\subsection{Interplays: deterministic-stochastic, inertia and uncertainty}

Only two of these six dimensions have a clear cut one-sided effect on the balance between early or deferred abatement whatever the framework adopted: these are Discounting and Risk aversion. For the remaining four, that is Inertia, Technical Progress, Irreversibility and Dependent Learning, the situation is more complicated. A more integrated analysis, such as Table 1 aiming at assessing the relative importance of the different effects enables to understand why in a stochastic framework, "Act more \& sooner" has more support than the opposite view.

In such a framework indeed some parameters have a Janus role: inertia for example inhibits early action but acts as a cost multiplier if, in case of bad news about climate change, the abatement has to be accelerated; in the same way, because technical change is driven by long standing efforts, waiting for a clear appreciation of climate damages is to take the risk of acting too late. The key problems stem in fact from the interplay between inertia and uncertainty: this is only because of the inertia of our economic system that uncertainty matters: without inertia, switching from one emission path to another would be costless and, in a perfect foresight setting, inertia would simply be one technical parameter among others.

We explored the role of this interplay in (Ha-Duong et al. 1997): selecting the same discount rate and autonomous technical progress coefficient as in models used to support (Wigley et al. 1996), this confirmed that the least cost abatement path towards a $550 \mathrm{ppm}$ concentration target should be only $2 \%$ below the baseline emissions in 2020 ; but this optimal departure jumps up to $14 \%$ if this $550 \mathrm{ppm}$ is the expected value of equiprobable 450, 550 and $650 \mathrm{ppm}$ ceiling, and if the uncertainty about what is the sustainable ceiling is resolved only

\footnotetext{
${ }^{2}$ The certainty equivalent $\mathrm{A}$ of a lottery $\tilde{c_{2}}$ for an utility function $v$ is defined by $v(A)=\mathrm{E} v\left(\tilde{c_{2}}\right)$
} 


\section{WHEN AND HOW MUCH MITIGATE CLIMATE CHANGE?}

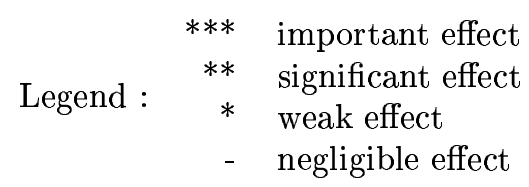

Discounting

\begin{tabular}{|c|c|c|c|c|}
\hline \multicolumn{5}{|c|}{ Inertia } \\
\hline$* * *$ & anticipate long effort & $\leftarrow \rightarrow$ & continuity & $* *$ \\
\hline \multicolumn{5}{|c|}{ Technical change } \\
\hline$* * *$ & autocatalyse & $\leftarrow \rightarrow$ & preparation $\neq$ action & $* * *$ \\
\hline \multicolumn{5}{|c|}{ Irreversibility effect } \\
\hline$* * *$ & cumulative pollution & $\leftarrow \rightarrow$ & sunk costs & $* *$ \\
\hline \multicolumn{5}{|c|}{ Dependant learning } \\
\hline$* *$ & technical feasability & $\leftarrow \rightarrow$ & slower danger signal & $*$ \\
\hline \multicolumn{5}{|c|}{ Risk aversion } \\
\hline$* *$ & extreme events & $\leftarrow \rightarrow$ & - & - \\
\hline & $\Downarrow$ & & $\Downarrow$ & \\
\hline & Act more \& sooner & & Act less \& later & \\
\hline
\end{tabular}

Table 1: This table summarises six key aspects of the timing debates. For each line, we assessed how much that aspect was an argument for early action (in the left column) and for delay (right column). Discounting, for example, is an important argument for delay, whereas we judged risk aversion as a significant argument for early action. The other four aspects are two-sided, as discussed in the text. 


\begin{tabular}{crlrl} 
Base year & \multicolumn{2}{c}{ Observed } & $\begin{array}{c}\text { Projected } \\
2010\end{array}$ \\
1990 & 1995 & & \\
& & & 873 & $-8 \%$ \\
949 & 936 & $-1 \%$ & $-6 \%$ \\
2086 & 2254 & $+8 \%$ & 1961 & $-6 \%$ \\
1311 & 925 & $-29 \%$ & 1298 & $-1 \%$ \\
& & & & \\
4346 & 4115 & $-5 \%$ & 4132 & $-5 \%$ \\
1774 & 2225 & $+25 \%$ & 4007 & $+126 \%$ \\
& & & & \\
6120 & 6340 & $+4 \%$ & 8139 & $+33 \%$
\end{tabular}

Table 2: Emissions of $\mathrm{CO}_{2}$ (Mt C per year), after Bolin (1998), and variations relative to the 1990 base year. Deforestation not included. Projections for Annex I parties according to the Kyoto protocol, for non Annex I parties assuming a $4 \%$ annual growth rate in emissions.

in 2020. In this case indeed the decision-making problem is to balance between the costs of switching towards a tighter target in 2020 and those of a too strict a pathway before 2020 if the ultimate target proves to be $650 \mathrm{ppm}$.

Observing the costs of a 20-year delay in action can easily highlight the reason for earlier abatement in a stochastic framework. These prove to be modest if the optimal target appear to be $550 \mathrm{ppm}$, but really significant for $450 \mathrm{ppm}$ : consequently, the costs of switching to 450 ppm too late dominate the costs of too early abatement if the optimal target happen to be $650 \mathrm{ppm}$. Unsurprisingly, this effect is all the more important as the resolution of uncertainties comes later: the optimal departure jumps up to $20 \%$ if full information comes in 2035. This effect is strongly correlated with the degree of inertia: doubling the degree of inertia results in an increase of the cost of delay from $14 \%$ to $35 \%$. Conversely costs of delay become negligible for capital life duration below 10 years, which is unrealistic.

\section{The Kyoto protocol in a public economy perspective}

\subsection{Tentative assessment of the quantitative tar- gets}

Kyoto targets, broadly recalled in Table 2, are interpreted in very opposite ways by various stakeholders: in the US, the opponents to any mitigation policy argue that they result from a pure political bargain and will entail dramatic costs for society; symmetrically, many environmental non governmental organisations express the concern that 


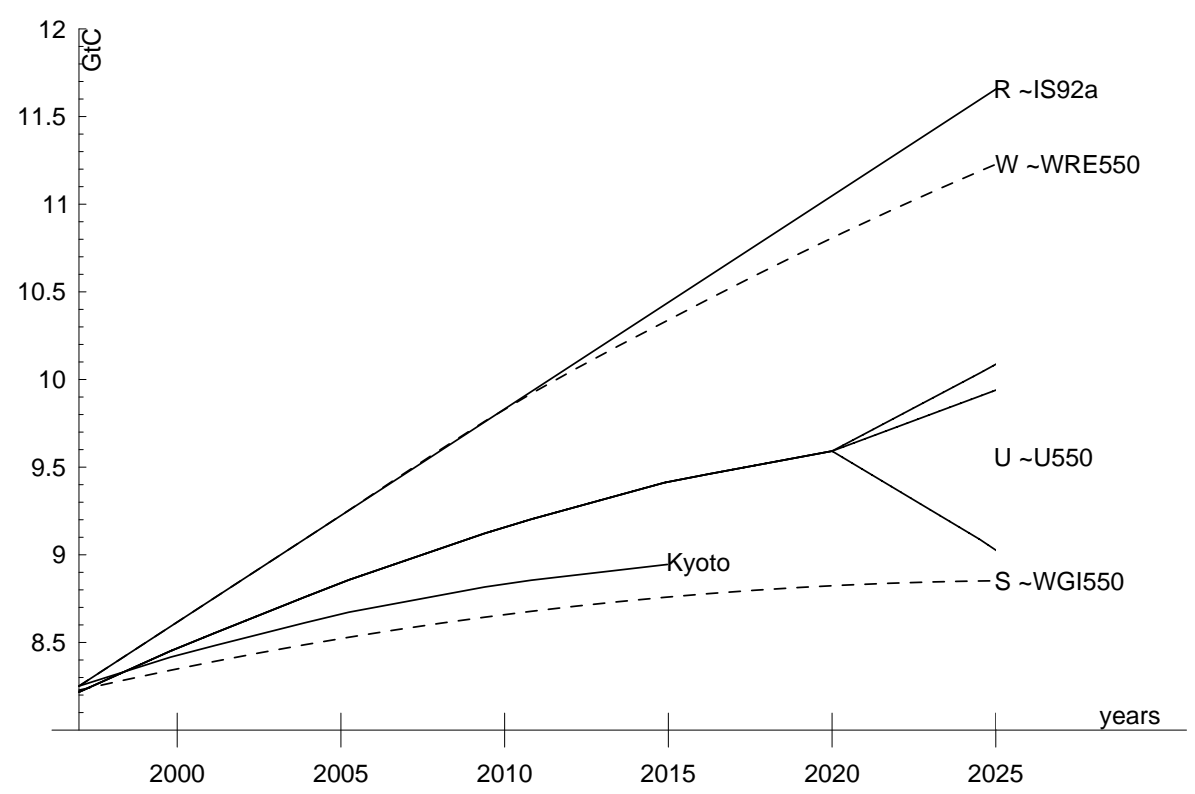

Figure 1: Different future $\mathrm{CO}_{2}$ emissions strategies, none of which is inconsistent with a $550 \mathrm{ppm}$ long term target. K stands for Kyoto, $\mathrm{R}$ for Reference, W for Wigley Richels and Edmonds, $S$ for Stabilisation computed after WGI methodology and $U$ stands for Uncertainty.

the adopted targets may not ambitious enough to enforce a real precautionary principle vis-à-vis climate risks.

Figure 1 compares the $\mathrm{CO}_{2}$ emissions trajectory corresponding to the Kyoto targets (curve labelled K) to different strategies proposed in the timing debate. First one can see that the emission Kyoto profile is well below the reference emission profile $\mathrm{R}$ following IS92a. This reference profile does not match the observed evolution for 1990-1995 Table 2: actual emission growth has been less than described in the IS92a scenario; but ongoing revisions of the IPCC scenarios suggests that the 1990-1995 time period may be very specific (among other reasons the economic collapse of ex centrally planned economies) and that business as usual emissions for Annex I may steadily increasing over the IS92 scenario. Thus, Kyoto targets do represent a significant departure from likely trends.

Second, the $\mathrm{K}$ curve is also well below the $\mathrm{W}$ curve, defined in (Wigley et al. 1996) by following the R path until 2010 and stabilising $\mathrm{CO}_{2}$ concentrations at the $550 \mathrm{ppm}$ level. Admittedly, that $\mathrm{W}$ path is closer to an economically rational emission path towards a $550 \mathrm{ppm}$ target than the $\mathrm{S}$ trajectory. The latter is defined here as in (Enting, Wigley \& Heimann 1994), by using a inverse carbon cycle model to find the smooth emission path consistent with an given concentration profile leading to stabilisation at $550 \mathrm{ppm}$.

Third, the $\mathrm{K}$ curve is also below the $\mathrm{U}$ curves which represents an optimal precautionary strategy given that the ultimate concentration 
ceiling is determined only in 2020 between 450,550 and $650 \mathrm{ppm}$. This $\mathrm{U}$ curve taken from (Ha-Duong et al. 1997) corresponds to a subjective probability distribution weighting equally the three ceilings.

This gives a first interpretation of the Kyoto targets: the position of the $\mathrm{K}$ curve below $\mathrm{U}$ curve suggests that more weight was implicitly given to $450 \mathrm{ppm}$ than to $650 \mathrm{ppm}$, as if policy-makers had retained an option value for preserving the environment and accounted for environmental irreversibility, technological dependent learning and risk aversion. In this sense, Kyoto targets can be seen as rather ambitious and revealing a real attempt to translate a precautionary approach in the face of unknown risks.

A step further can be made in a cost-benefit framework to reveal what implicit beliefs about climate damages corresponds to Kyoto targets. To date, many integrated assessment models have been led to use some kind of damage function in order to represent how the climate damages (in \% of Gross World Product, for example) increase with the rise in atmospheric $\mathrm{CO}_{2}$ concentration (in ppm). For example, damage as a function of concentration $c$ could be computed $\mathrm{as}^{3}$ :

$$
\text { damage }=d \times\left[\Delta T_{2 x} \frac{\log (c / 278)}{\log (2)}\right]^{2}
$$

This kind of damage functions are not uncommon in the litterature. Here we would like to point out two serious dynamic consistency issues arising with them when it comes to analysing climatic policies in a stochastic decision making framework. These are illustrated Figure 2A (top panel).

First, the easiest parameters to adjust when one needs to study the possibility of extreme damages are $d$ (the damage scale) and $\Delta T_{2 x}$. For example, the EMF14 uncertainty subgroup study suggested, following Nordhaus (1995), to increase $\Delta T_{2 x}$ by $2.5^{\circ} \mathrm{C}$ and to multiply $d$ by 7.8 , as shown on the figure. This may be inconvenient, as it leads to an average damage function lying well above the base case. Consequently, results under uncertainty can not be compared to results in the base case, since the expected damage is different.

The second inconsistency, more difficult to workaround, it is that the catastrophic High case and the Hclimate cases appear incompatible with the fact that presently, no or low damages can be attributed to anthropogenic climate change. This raises the issue of the relationship between assumptions on the shape of the damage function and the date of resolution of uncertainties.

This is why the implicit damage functions corresponding to Kyoto appear to be much more non linear than the ones above. For example, they could belong to the following familly ${ }^{4}$, illustrated in figure $2 \mathrm{~B}$ (bottom panel):

\footnotetext{
${ }^{3}$ With $\Delta T_{2 x}=2.5^{\circ}$, the bracketed term is temperature increase $\Delta T$. The overall damage scale $d=1.33 / 9$ can be calibrated after DICE damage function so that a $3^{\circ}$ increase in temperature results in $1.33 \%$ damage.
} 

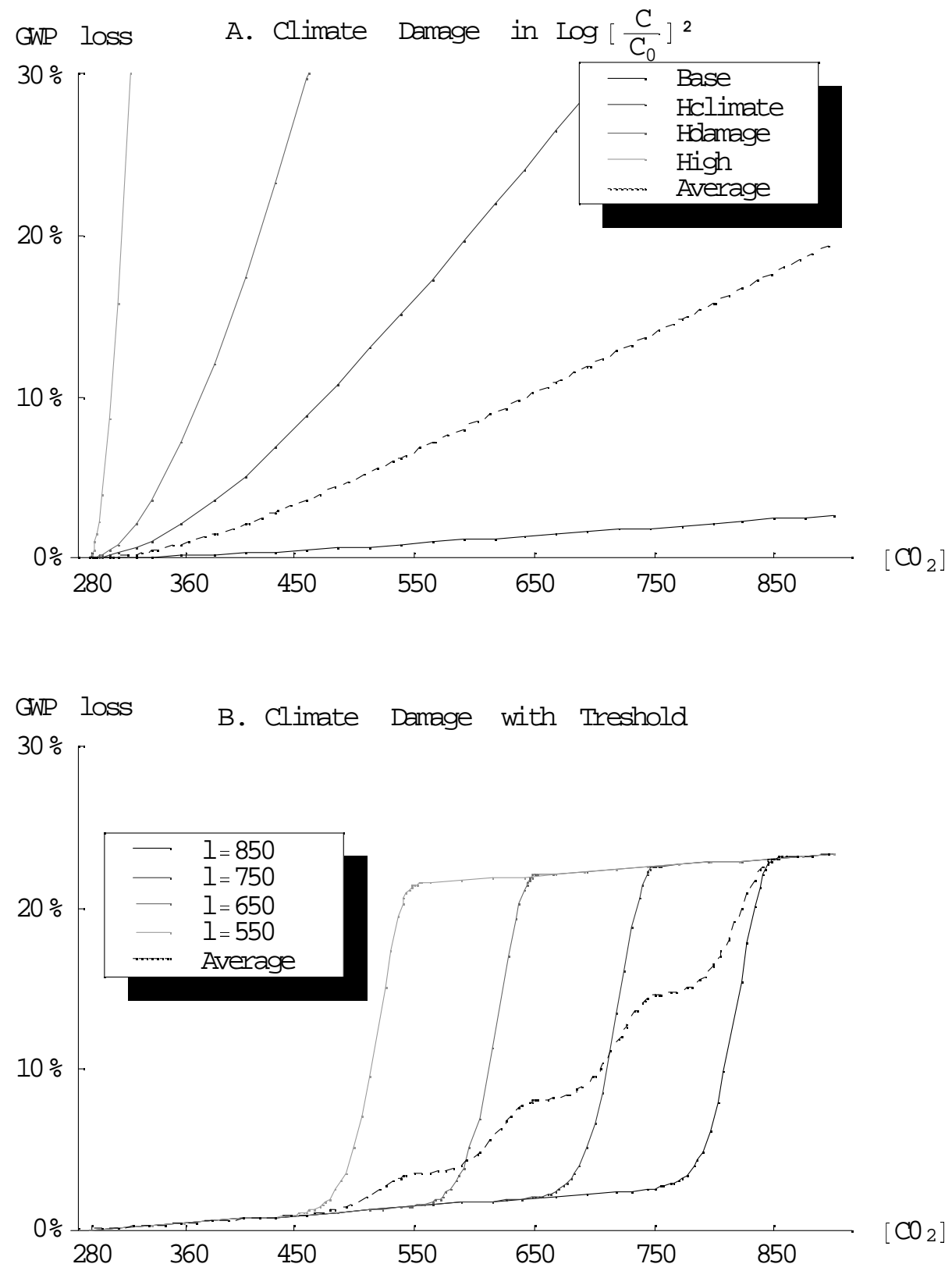

Figure 2: Distribution of climate damage as a function of $\mathrm{CO}_{2}$ concent ration, for five states of the world: 1 . base case; 2. High climate sensitivity $\left(+2.5^{\circ} \mathrm{C}\right), 3$. High damages $(\times 7.8), 4$. catast rophic High case (combination of 3 and 4 ), and 5 . average. Top panel A. uses quad ratic functions and follows No rdaus (1995). Bottom panel B. shows damage functions with a concent ration th reshold and ceiling. 


$$
\text { damage }=1.5(c / 278-1)+30 e^{-\left(2 \frac{c-l}{80}\right)^{2}}
$$

To illustrate the importance of non linearity in the damage function, we conducted in (Ha-Duong 1998a) numerical experiments with the DIAM model. We computed optimal $\mathrm{CO}_{2}$ abatement in 2020 under uncertainty for two hypothesis on the shape of the damage function.

- First, using equation 2, we assumed that the scale of the damage function would be known only in 2020. It appeared that the effect of uncertainty was negligible in front of discounting and inertia.

- Then, using equation 3 , uncertainty was set on the parameter $l$ , which represents the level at which marginal climate damages start to increase abruptly. Under these assumptions, the optimal abatement for a deterministic resolution was in the $4 \%-11 \%$ interval, whereas in stochastic mode the optimum reduction ranged from $14 \%$ to $23 \%$ of 2020 emissions.

This allows to suggest that, in a cost-benefit framework, Kyoto quantitative targets are more consistent with the idea that climatic damages could increase abruptly and non-linearly.

\subsection{Dynamic inconsistencies behind the Flexibility Mechanisms?}

Together with quantitative targets, the Kyoto Protocol contains several provisions for the use of 'flexibility mechanisms' to facilitate every Annex B country to keep within its emissions assignment: the emissions trading amongst Annex B countries, the joint implementation projects in Annex B countries and the Clean Development Mechanisms in the non Annex B countries. In addition, the existence of the so-called European Bubble lead to open the possibility for other Annex B countries to create new 'bubbles' within which they can renegociate their assignments through a political deal between GHG emissions and other economical and political objectives.

A naive view of the 'when flexibility' mechanisms related to the timing debate leads to the idea of banking or borrowing emission allowances from one period to another. The Kyoto Protocol rules out the possibility of borrowing, as such a possibility would have indeed undermined the credibility of the Protocol: any government may be tempted to resort to borrowing to avoid adopting unpopular measures and to pass the burden to its successors. In the absence of credibility of important sanctions at the international level if the level of borrowing

\footnotetext{
${ }^{4}$ This specification is valid as long as concentration $c$ is lower than the ceiling $l$. The linear part is scaled so that doubling of preindustrial concentration results in 1.5 points of damage, the non-linear part is scaled so that damages jump suddenly by $30 \%$ of GNP over a concentration range of $80 \mathrm{ppm}$. In the figure, probabilities for 850,750,650 and 550 ceiling are respectively $40 \%, 30 \%, 20 \%$ and $10 \%$. Note that for these damage functions, the critical parameter is not the ceiling $l$ at which the damage is $30 \%$ of GNP, but the threshold $l-80 \mathrm{ppm}$ at which non linearities begin to be significant.
} 
becomes too high, the ruling out of borrowing was the only way the avoid such a risk.

However, a more dynamic and systemic view of these flexibility mechanisms brings back to the timing issue because of possible dynamic inconsistencies between decisions and behaviours before and after the end of the first budget period (2012). These risks are far from being purely theoretical and originated a burning debate between the European Union, the US and the other countries grouped in the JUSCANZ (Canada, New-Zealand, Australia, Japan), about the articulation between so-called policies and measures (P\&M) and flexibility mechanisms, principally emissions trading systems. Article 17 of the Kyoto Protocol states (our emphasis):

The Parties included in Annex B may participate the emissions trading for the purposes of fulfilling their commitments under Article 3. Any such trading shall be supplemental to domestic action for the purpose of meeting emission limitation and reduction commitments under that Article.

This phrasing results from a compromise due to the insistence of the European Union that domestic P\&M should be the main means for every country to meet its own target. In March 1998, the European Environment Council made a step further and demanded that the forthcoming Conference of the Parties at Buenos Aires place a "concrete ceiling" upon the use of all flexibility mechanisms ${ }^{5}$. At the SBSTA meeting at Bonn (2-12 June 1998) a non-paper circulated by the UK on behalf of the European Community and its member states plus the Czeck Republic, Slovakia, Croatia, Latvia, Switzerland, Slovenia, Poland and Bulgaria states: "We believe that domestic actions should provide the main means of meeting commitments under Article 3".

The economic rationale for such a demand may appear unclear for economic analysts who reason in a first best world. Indeed, if a GHG trading system is set up, an international price of carbon will emerge and every government will take those $\mathrm{P} \& \mathrm{M}$ whose marginal cost is lower than this price. In this sense, a trading system cannot be but supplemental to domestic action. The European demand can then be presented as paradoxical: to limit the access to cheap abatement potentials will increase the overall cost of climate policies, and the incremental burden will be higher for countries with high marginal abatement costs than for countries for low marginal costs, consequently it will penalise more the European countries than the US.

But this static reasoning is challenged by the very factors which underpinned the timing debate before Kyoto: inertia, technical change and uncertainty. The bottom line of the argument is that, if it is true that a well designed trading system minimises the abatement costs at each point in time in a given technical endowment, there is no

\footnotetext{
${ }^{5}$ The form of this concrete ceiling has not been precised so far; some interpret it as a quantitative limit of the assigned amounts which can be acquired through trading, others as a set of criteria ensuring that the 'supplementary to' condition is fulfilled.
} 
garantee that it minimises the costs of climate policies over the long run. Here again the logical distinction between abatement and policy signals come into play as well as the time lag between signals and technical responses in the presence of inertia and uncertainty about long term trends.

One of the lesson of the debate resumed in the first section is indeed that price-signals cannot be the sole driver of technical change. The question is how to articulate necessary price signals (in the form of carbon tax or through trading systems) with other policy tools to trigger an endogenous process where learning by doing, increasing returns to adoption and network externalities come into force. This type of problems may arise in any sector of the economy but becomes critical for the transportation sector which generates the most important upward increase of carbon emission in Annex B countries.

This risk is aggravated by the fact that because of the existing level of taxation for motor fuels in many countries, only very high prices of carbon will result into a significant curbing down of emissions from the transportation sector. And such a perspective is unlikely during the first budget period because of the possible discovery of important negative costs potentials in the Annex B countries and, more importantly because of the existence of fictitious reductions in Russia and Ukraine or from projects carried out under the Clean Development Mechanism. Given the economic advantage of existing oil-based motorfuels, it is unlikely, or at least dubious, that prices of carbon given by current modelling exercises (between 40 to $120 \$ / \mathrm{t}$ ) will be apt to trigger large scale substitution to conventional gasoline. This is all the more uncertain if all the external costs and general equilibrium impacts of substitutes such as biofuels or electric cars are to be considered in the balance.

Consequently, the risk exists that many countries will meet no macroeconomic obstacle in importing permits during the first budget period and could undertake no efforts on the structural trends in the transportation sectors through infrastructure policies and urban planning, and to modify the life-styles. Some countries may then be locked in a very carbon demanding development pattern and any attempt to adopt more ambitious targets beyond 2012 may then be proven politically and economically difficult.

The following numerical exercise aims at demonstrating the orders of magnitude at stake.

\section{An quantitative assessment of possible dynamic inconsistencies}

To show out the possible dynamic inconsistencies which justify the EU "supplemental to" condition for trading systems, it matters to capture the heterogeneity of inertia of emission activities. The model STARTS (Sectoral Trajectories with Adaptation and Response Turnover of Stocks) developed by Lecocq, Hourcade \& Ha-Duong (1998) is an intertempo- 
ral dynamic optimisation model, such as DIAM, but with two sectors characterised by different inertia levels. STARTS includes an explicit description of capital stock generations.

Renewing a capital vintage before the end of its economic life duration results in a loss. The central difference with classical growth model using an aggregate capital value and turnover rate is that STARTS is able to fully explicit the trade-off between leap-frogging and accelerated turnover abatement. Given an emission reduction target, there is indeed a choice between buying very low emitting capital stock or scrapping some vintages before the end of their economic life to replace them with medium emitting capital.

For example, an economy replacing $25 \%$ of its capital every decade will be obliged to adopt a zero emission technique (let us say one solar plant) if it is committed to cut $25 \%$ of its emissions. Now would the cost of such a technique be very high, it might be cost effective to replace one additional capital vintage and build two gas plants saving each $12.5 \%$ of previous emissions. The optimal trade-off requires the marginal saving on the abatement costs to be equal to the costs of scrapping equipment prematurely.

In STARTS, each sector encompasses both the capital stock driving the energy demand and the corresponding energy supply but they are characterised with different capital life duration. The rigid sector covers transportation infrastructures (roads, airports or railways) and the part of urban planning which shapes urban patterns and transportation needs within cities and is supposed to have a 60 years mean capital duration. The flexible sector covers housing and industry with a 30 years capital duration. The reason for this gross classification is that we chose to place the focus upon transportation and urban infrastructures which have a far longer capital life duration than any other kind of capital (buildings excepted) and, more importantly, give rise to typical self reinforcing loops between supply and demand which upgrade the inertia of the economic system.

Using a central emission baseline based on the IPCC IS92a scenario and sectoral emission projections from IIASA (1995), STARTS was used to generate optimal abatement paths for three concentration ceilings (450, 550 and $650 \mathrm{ppm}$ ) and two hypothesis on the starting date of abatement programs (2000 or 2020). Then we duplicated these simulations with a $25 \%$ higher emission growth rate in the transportation sector. The emission growth rate in this sector thus rises from 2 to $2.5 \%$ annually. This assumption is consistent with the uncertainties surrounding the future transportation systems, particularly in the developing countries.

Two results of interest can be drawn out of these simulations:

- First, as shown Figure 3 the model's response to delayed action proves highly non linear with concentration. With concentration ceilings above $550 \mathrm{ppm}$, the optimal abatement policy does not change much with or without a 20 -year delay of action. But with a $450 \mathrm{ppm}$ targetn a 20-year delay results in a heavy acceleration of abatement in the flexible sector (i.e. mostly the industrial 


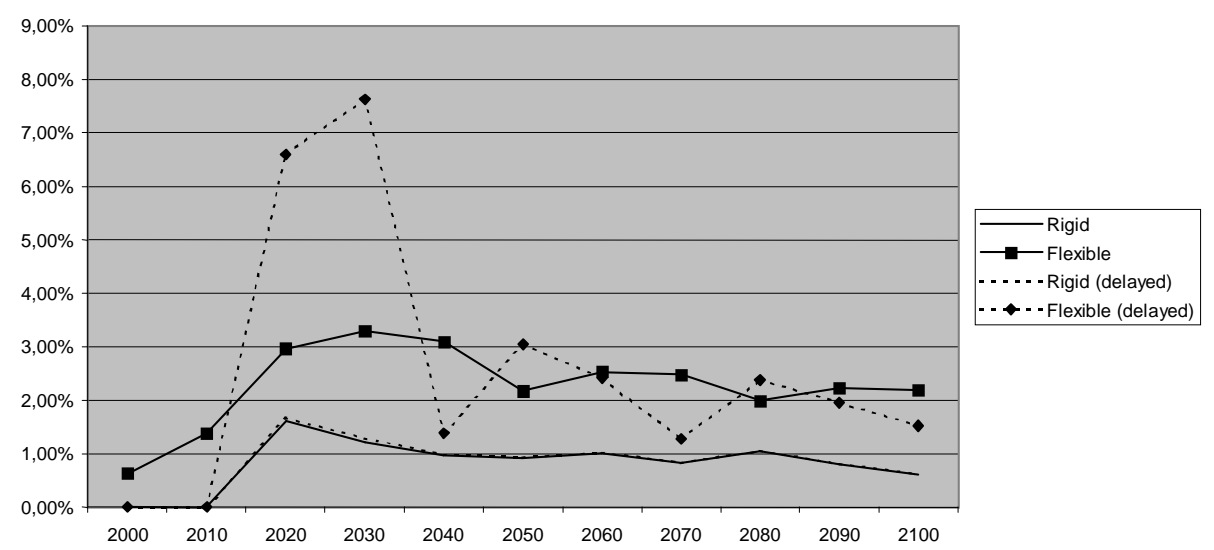

Figure 3: The 'cost surge effect' falling upon the flexible sector in case of a 20 year delay of action (target: $450 \mathrm{ppm}$ ).

sector).

- Second, a rise in the rigid sector's growth rate yields higher abatement levels in the rigid sector (see figure 4). The reason for this response is easily explainable since higher emission trends reduce the margins of freedom in the rigid sector. The important point is the order of magnitude of such a change: optimal abatement levels in the rigid sector in 2020 rise significantly from $8 \%$ to $18 \%$ at $550 \mathrm{ppm}$. Furthermore, a 20-year delay of action, which was previously negligible at such a ceiling, becomes strongly significant. This confirms that the growth rate of the rigid sector matters critically for short term policy.

The policy implication of the above results is straightforward; they highlight the importance of early action in the rigid sector by showing how costly any delay in the abatement or any underestimation of emission growth rate in this sector might be. They confirm that, serious dynamic inconsistencies of choice may occur if price signals from trading systems in the first budget period, is not sufficient to trigger abatement action in transportation or housing. The orders a magnitude of the penalty incurred to counterbalance this effect is so high that some Parties will renounce to adopt tighter targets for the second budget period. This is why policy measures in urban planning and modal structures are required to curve emission trends in rigid sectors and thus reinforce and back up the market process.

\section{Conclusion: perspectives for the "sup- plemental to" debate}

The judgments that can be made about the result of Kyoto Conference in a sustainable development perspective are somewhat contradictory. 


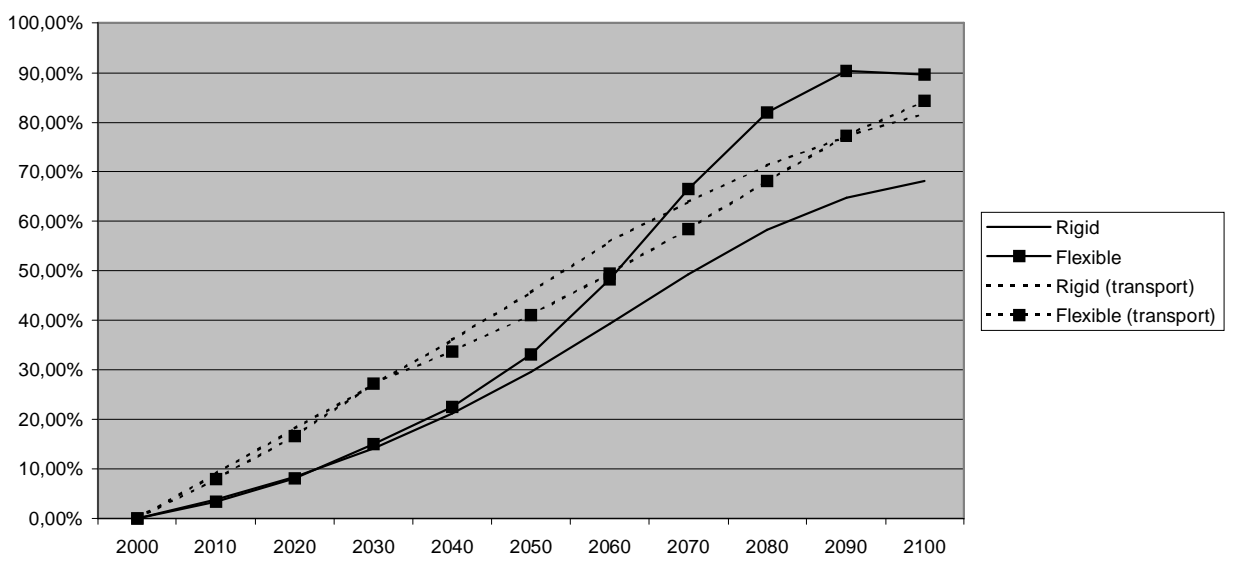

Figure 4: Abatement level. Dotted lines correspond to a higher reference path in the transportation sector.

On one hand, Kyoto emissions targets are economically consistent with an emission path towards a $550 \mathrm{ppm}$ concentration ceiling, but with the interest of preserving the option of shifting towards a $450 \mathrm{ppm}$ if future advances in climate science or climate surprises suggest that it would be wise to stay below such a ceiling. The departure from current emissions trends is significant and, in contradiction to some polemic judgments, it cannot be said that Kyoto does not represent a serious consideration of climate issue.

But on the other hand, if one looks at the dynamics behind the aggregate figures, there is no certainty that, while respecting their emissions assignment, Annex B countries will not be trapped in emission paths which will make it difficult to adopt tighter abatement targets beyond 2012. Behind what technically appears as a dynamic consistency problem due to the dynamics in rigid sectors such as transportation or housing, lies in practice the sensitive issue of the long term innovation and of the evolution of life styles. If domestic policies and measures are not adopted in due time in Annex B countries, the emergence of GHG trading systems may then create a masking effect which will end into a failure of climate policies.

This risk is all the more important that the entry of developing countries within the Annex B will be necessary after 2012. Because of the non observability of emissions baselines, any such entry may indeed generate a new wave of excess assigned amounts, resulting in low carbon prices over the long run and in the absence of incentive to curb down real emissions trends. The problem is that the reflex of imposing quantitative limits to emissions trading cannot be an appropriate response to such a challenge.

Beyond the intrinsic perverse effects of such a limitation (higher trading rent for countries with excess assigned amounts, higher burden on countries with higher marginal costs, possibility of transforming 
the US in carbon exporters), this approach does not take enough into consideration the logical distinction between timing of action and timing of the abatement, and the fact that an aggregate emissions target is not a good measure of ongoing efforts. For example a country launching a drastic restructuring of its transportation system or reshaping its stock of building to lower the heating and air conditioning requirements will have far less positive performances over one two decades than a country shifting quickly its electric generation system from coal to gas while taking no measure to control the increase of its domestic demand over the long run.

These contradictions trace back to an underestimation of the "price versus quantities" dilemna which undepins the climate policies coordination process (Pizer 1997).

There were at the outset of the nineties two competing approaches to climate negotiation. The first was to try and co-ordinate policies and measures without pretending to set up quantitative targets ; the second was to set up targets. The latter was finally adopted because it provided to environmental NGO an apparent level of guarantee of action, while avoiding in practice the difficult debate about the coordinated policies and measures. But the mix of medium term targets and flexibility mechanisms adopted at Kyoto in turn does not guarantee that the ultimate objective will be fulfilled.

This consideration, plus the difficulty of embarking developing countries into the adoption of quantitative targets without assigning them emission amounts above their baseline (Jacoby, Prinn \& Schmalensee n.d.), should lead to reconsider, for the period beyond 2012, an approach in terms of co-ordinated policies and measures (including higher energy prices) as the only real guarantee of achieving the objective of the FCCC. Could this be done within the framework of the Convention and the Protocol, since to re-negociate it would certainly be politically impractical ? Our opinion is that the phrasing of the Protocol is flexible enough for this. Recognition of emission trading facilitates compromise with the heavy industries exposed to international competition. At the same time, nothing is said for the second budget period. This opens an opportunity for placing again the focus upon coordinated policies and measures, aiming to curb current polluting trends in life-styles and technical choices. After all, more than two-third of carbon emissions comes from sectors of the economy non-exposed to international competition.

\section{References}

Alcamo, J., ed. (1994), IMAGE 2.0: Integrated Modeling of Global Climate Change, with papers by The IMAGE Project, Kluwer Academic Publisher. Reprinted from Water, Air, and Soil Pollution $76(1-2)$.

Arthur, C. W. (1989), 'Competing technologies, increasing returns and lock-in by historical trends', Economic Journal 99(394), 116-131. 
Azar, C. (1998), 'The timing of $\mathrm{CO}_{2}$-emissions reduction - the debate revisited', International Journal of Environment and Pollution 10.

Bolin, B. (1998), 'The Kyoto negotiations on climate change: A science perspective', Science 279(5349), 330-331.

Bruce, J. P., Lee, H. \& Haites, E. F. (1996), Climate Change 1995 - Economic and Social Dimensions of Climate Change, Contribution of Working Group III to the Second Assessment Report of the Intergovernmental Panel on Climate Change, Cambridge University Press, Cambridge, Massachussets.

Dixit, A. K. \& Pindyck, R. S. (1994), Investment under uncertainty, Princeton University Press, Princeton, New Jersey.

Enting, I., Wigley, T. \& Heimann, M. (1994), Future Emissions and Concentrations of Carbon Dioxide: Key Ocean/Atmosphere/Land Analyses, number 31 in 'Division of Atmospheric Research Technical Paper', CSIRO, Australia. Also published on the internet, formatted as Postscript, on CDIAC electronic archives, http://cdiac.esd.ornl.gov/ndps/db1009.html, accessed 9/7/98.

Fagan, M. N. (1997), 'Resource depletion and technical change: U.S. crude oil finding costs from 1977 to 1994', Energy Journal 18(4), 91-105.

Fisher, A. C. \& Hanemann, W. M. (1987), 'Quasi option value: Some misconceptions dispelled', Journal of Environmental Economics and Management 14, 183-190.

Grubb, M. J. (1997), 'Technologies, energy systems and the timing of $\mathrm{CO}_{2}$ emissions abatement', Energy Policy 25(2), 159-172.

Ha-Duong, M. (1998a), Comment tenir compte de l'irréversibilité dans l'évaluation intégrée du changement climatique ?, $\mathrm{PhD}$ thesis, École des hautes Études en Sciences Sociales, Paris.

Ha-Duong, M. (1998b), 'Quasi-option value and climate policy choices', Energy Economics pp. 1-23. Forthcoming. Conference version published on the internet, formatted as Portable Document File, on the global Network of environmental economists working paper library, subject area 9-Economic Theory and the Environment, http://www.feem.it/gnee/pap-abs/haduong.pdf, accessed $9 / 7 / 98$.

Ha-Duong, M., Grubb, M. J. \& Hourcade, J.-C. (1997), 'Influence of socioeconomic inertia and uncertainty on optimal $\mathrm{CO}_{2}$-emission abatement', Nature 390, 270-274. Also published on the internet, formatted as Portable Document File, as part of the Nature $W e b$ Specials on the Kyoto Climate Conference, accessed 9/7/98.

Hourcade, J.-C. (1993), 'Modelling long-run scenarios: methodology lessons from a prospective study on a low $\mathrm{CO}_{2}$ intensive country', Energy Policy 21(3), 309-326.

Hourcade, J.-C. \& Chapuis, T. (1995), 'No-regret potentials and technical innovation.', Energy Policy 23(4/5), 433-445. 
IPCC (1995), Second Assessment Synthesis of Scientific-Technical Information Relevant to Interpreting Article 2 of the UN Framework Convention on Climate Change, UNEP/WMO. Also available electronically from IPCC website.

IPCC, Working Group I (1997), Stabilisation of Atmospheric Greenhouse Gases: Physical, Biological and Socio-economic Implications (IPCC Technical paper III), UNEP/WMO.

Jaccard, M. (1997), Heterogenous capital stock and decarbonating the atmosphere: does delay make cents ? Personal communication.

Jacoby, H. D., Prinn, R. G. \& Schmalensee, R. (n.d.), 'Kyoto's unfinished business', Foreign Affairs 77(4), 54-66.

Kolstad, C. D. (1994), The timing of $\mathrm{CO}_{2}$ control in the face of uncertainty and learning, in E. van Ireland, ed., 'International Environmental Economics', Elsevier, Amsterdam, chapter 4.

Lecocq, F., Hourcade, J.-C. \& Ha-Duong, M. (1998), 'Decision-making under uncertainty and inertia constraints: sectoral implications of the when flexibility', Energy Economics This issue, 18 p.

Mégie, G. (1992), La modélisation en climatologie et physico-chimie de l'atmosphère: le statut du long terme et le jeu des incertitudes, in 'Séminaire ECLAT-ESCG-PRISTE "Environnement et Développement durable : un débat interdisciplinaire"', Centre National de la Recherche Scientifique, Paris.

Narain, U. \& Fisher, A. C. (1998), Irreversibility and catastrophic global warming, in 'World Congress of Environmental and Resource Economists', Venice, Italy. Deposited electronically at the GNEE archive.

Nordhaus, W. D. (1995), Notes on scenarios for uncertainty subgroup. Letter to the participants of the EMF 14.

Pizer, W. A. (1997), Prices vs. quantities revisited: The case of climate change, Discussion Paper 98-02, Resources for the Future. Available electronically at www.rff.org as 9802.pdf. Accessed 3/10/98.

Schelling, T. (1995), Keynote speech to the international energy workshop, IIASA, Laxenburg, Austria.

Schneider, S. H. \& Goulder, L. H. (1997), 'Achieving low-cost emissions targets', Nature 389, 13-14.

Toth, F. L., Bruckner, T., Füssel, H.-M., Leimbach, M. \& PetschelHeld, G. (1997), The tolerable window approach to integrated assessments, in 'IPCC Asia-Pacific Workshop on Integrated Assessment Models'.

UNF (1992), 'United nations framework convention on climate change', Full text of the Convention and the Kyoto Protocol are available at the UNFCCC secretary website http://www.unfccc.de/. Accessed $2 / 8 / 98$.

Wigley, T. M. L., Richels, R. \& Edmonds, J. A. (1996), 'Economic and environmental choices in the stabilization of atmospheric $\mathrm{CO}_{2}$ concentrations', Nature 379(6562), 240-243. 